\title{
Multiple Description Source Coding for Cognitive Radio Systems
}

\author{
Husheng Li
}

\begin{abstract}
Multiple description coding (MDC) is applied for delay-sensitive data traffic to alleviate interruptions caused by primary users in cognitive radio systems. For Gaussian source, a simplified algorithm for the exhaustive search of optimal distortions and rates is proposed based on the achievable region of coding rates and delay requirement. For practical realtime image coding, source splitting is applied to generate multiple descriptions. When there is a single channel, two successive descriptions are composed of large and small coefficients in discrete cosine transform (DCT) domain, respectively. When there are two channels, even and odd coefficients in DCT domain are used to construct two parallel descriptions. Numerical results show that MDC can effectively combat the interruptions caused by primary users in cognitive radio systems.
\end{abstract}

\section{INTRODUCTION}

In recent years, a novel technology named cognitive radio has attracted intensive studies in the field of wireless communications and networking [11] [20]. The fundamental purpose of cognitive radio is to alleviate the underutilization of frequency spectrum by allowing users without license (called secondary users) to access licensed frequency spectrum when users with license (called primary users) are not present.

The new mechanism of opportunistic spectrum access brings a paradigm shift and many challenges for the design of wireless communication systems. Many studies have been carried out for spectrum sensing [5], i.e. looking for opportunities in frequency spectrum, and resource allocation, i.e. how to allocate available frequency spectrum to different secondary users. For the data transmission in physical layer, rateless coding has been applied to convey multimedia over cognitive radio links [9].

However, little work has been done for source coding in cognitive radio systems. A new feature in cognitive radio is that the data transmission of secondary users may be interrupted by primary users, thus incurring unreliability and delay. If the data traffic is elastic, such interruptions have little impact the source coding and thus traditional source coding techniques can still be applied. However, if the data traffic is delay-sensitive, source coding should be designed according to the pattern of interruptions, which brings a new challenge for cognitive radio systems.

In this paper, we consider delay-sensitive sources like realtime image transmission and apply the technique of multiple description coding (MDC) to combat the interruptions from

H. Li is with the Department of Electrical Engineering and Computer Science, the University of Tennessee, Knoxville, TN, 37996 (email: husheng@eecs.utk.edu). This work was supported by the National Science Foundation under grant CCF-0830451.

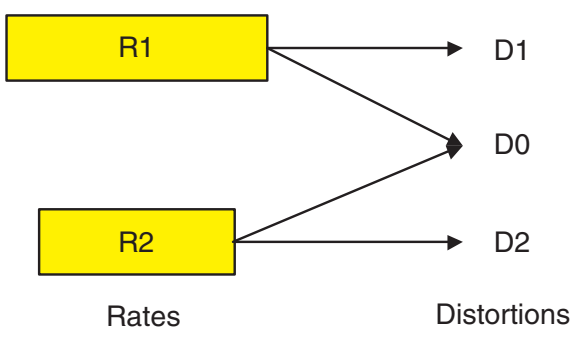

Fig. 1: Illustration of MDC.

primary users. The basic idea of MDC is to use multiple different descriptions (source codewords) for the same source. The distortion of source reconstruction can be reduced as more descriptions are received. A two-description case is illustrated in Fig. 1, in which two descriptions with coding rates $R_{1}$ and $R_{2}$ are used; if only one description is received, the corresponding distortion will be $D_{1}$ and $D_{2}$, respectively; if both descriptions are received, the distortion by combining the two descriptions will be $D_{0} \leq \min \left(D_{1}, D_{2}\right)$. MDC has been well studied in the community of information theory and bounds for the coding rates have been obtained for given distortion requirements [2] [3] [4] [16] [19]. MDC is also widely applied in practical source coding, e.g. speech coding [6], image coding [15] and video streaming [1] [10] [17] [21].

We will address the following challenges of applying MDC in cognitive radio systems:

- Rate-distortion optimization: there is a tradeoff between the rates and distortions, i.e. the smaller rates are (equivalently the larger probability that the descriptions can be received successfully), the larger the distortions are. We will define an utility function and turn the selection of rate and distortion into an optimization problem.

- Application in practical source coding: we will apply MDC in the source coding of realtime image.

The remainder of this paper is organized as follows. The system model is introduced in Section II. MDC with Gaussian source is discussed in Section III, where we consider both single-channel and multi-channel cases. In Section IV, MDC is applied in image coding over cognitive radio links. Numerical results and conclusions are provided in Sections V and VI.

\section{SySTEM MOdEL}

We consider the source coder of a secondary user illustrated in Fig. 2, where the source coder receives the channel properties passed from physical layer and the requirement 


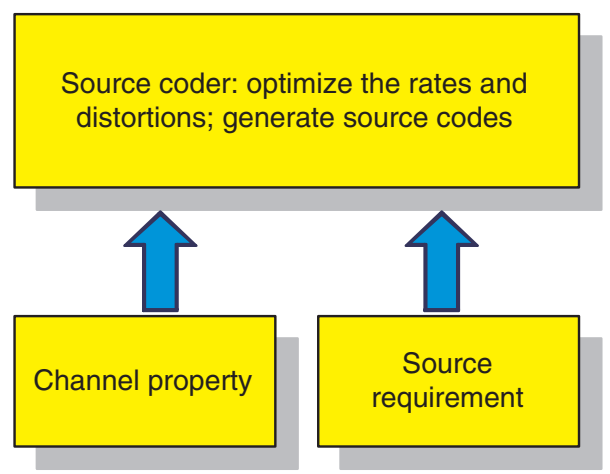

Fig. 2: System diagram of source coder.

from the source. Then, multiple different descriptions (source codewords) are generated and then transmitted.

The following assumptions are used throughout the paper for simplicity of analysis. They can be relaxed and the analysis can be extended to more general cases.

- Frequency channel properties are perfectly known. Each channel bandwidth is fixed when there is no active primary user.

- We consider perfect channel coding such that no channel decoding error occurs.

- Only interruptions from primary users are considered. Other negative factors like deep fading or strong interference are ignored.

- For Gaussian source, the occupancy of primary users over each frequency channel is modeled as a Markov chain, which has been assumed in many studies [20]. The state transition probability from busy (occupied by primary users) to idle (not occupied by primary users) is denoted by $P_{B I}$ and the probability from idle to busy is denoted by $P_{I B}$. For practical image source coding, we use the more practical semi-Markov chain to model the occupancy of primary users, i.e. the transition between the two states are not necessarily instantaneous and a distribution of transition period is used to explicitly model the duration of idle or busy states (in a Markov chain, the duration is geometrically distributed).

- There is a deadline for all codewords, denoted by $T_{d}$. Only descriptions fully received before the deadline can be used for the reconstruction of source.

\section{MDC FOR GAUSSIAN SOURCE}

In this section, we consider the MDC for Gaussian source, which is denoted by $X_{1}, X_{2}, \ldots, X_{n}, \ldots, X_{N}$ ( $N$ is the number of source symbols in each source block). The source random variables are independent and identically distributed (i.i.d.) with expectation 0 and variance 1 . We first introduce known results about MDC for Gaussian source and then apply them to both single-channel and multi-channel cases. For simplicity, we consider only two descriptions.

\section{A. Known Results About MDC for Gaussian Source}

For Gaussian source, it has been shown that, when $D_{1}+$ $D_{2}-D_{0} \leq 1^{1}$, the achievable region of coding rates is given by [3]

$$
R_{1} \geq \frac{1}{2} \log \frac{1}{D_{1}}
$$

and

$$
R_{2} \geq \frac{1}{2} \log \frac{1}{D_{2}}
$$

and

$$
R_{1}+R_{1} \geq \frac{1}{2} \log \frac{1}{D_{0}}+\frac{1}{2} \log Z
$$

where

$Z=\frac{\left(1-D_{0}\right)^{2}}{\left(1-D_{0}\right)^{2}-\left(\left(1-D_{1}\right)^{1 / 2}\left(1-D_{2}\right)^{1 / 2}-\left(D_{1}-D_{0}\right)^{1 / 2}\left(D_{2}-D_{0}\right)^{1 / 2}\right)^{2}}$

The shape of achievable region is illustrated in Fig. 3 (a).

\section{B. MDC in A Single Channel}

When there is only one channel, we assume that the two descriptions are sent in the order of description 1 first and then description 2. Therefore, only the following three situations can happen:

- Event 0: both descriptions are received and distortion $D_{0}$ is achieved.

- Event 1: only description 1 is received and distortion $D_{1}$ is achieved.

- Event 2: none of the descriptions is received.

We denote by $P_{0}$ and $P_{1}$ the probabilities of the first two events. Obviously, the MDC in single channel case is actually a progressive coding [12]. We consider the following expected utility

$$
J=P_{1} U_{1}\left(D_{1}\right)+P_{0} U_{0}\left(D_{0}\right),
$$

where $U_{1}$ and $U_{0}$ are the utility functions of the first two events.

It is prohibitively difficult to obtain an explicit expression for the rates and distortions optimizing the expected utility in (4) since $P_{1}$ and $P_{0}$ are dependent on $R_{1}$ and $R_{0}$. In this paper, we consider the asymptotic case of $N$ and $T_{d}$, i.e. $N, T_{d} \rightarrow \infty$ and $\frac{T_{d}}{N}=\alpha>1$, for simplicity of analysis. In the asymptotic case, we have

$$
P_{0}=\left\{\begin{array}{l}
1, \text { if } R_{1}+R_{2} \leq \alpha P_{I} \\
0, \text { if } R_{1}+R_{2}>\alpha P_{I}
\end{array},\right.
$$

where $P_{I}$ is the stationary probability that the channel is idle, which can be determined by the following equation

$$
\left(\begin{array}{c}
P_{I} \\
1-P_{I}
\end{array}\right)=\left(\begin{array}{cc}
1-P_{I B} & P_{B I} \\
P_{I B} & 1-P_{B I}
\end{array}\right)\left(\begin{array}{c}
P_{I} \\
1-P_{I}
\end{array}\right),
$$

and

$$
P_{1}=\left\{\begin{array}{ll}
1, & \text { if } R_{1} \leq \alpha P_{I} \text { and } R_{1}+R_{2}>\alpha P_{I} \\
0, & \text { otherwise }
\end{array} .\right.
$$

${ }^{1}$ When $D_{1}+D_{2}-D_{0}>1$, the distortion is high and the achievable region is trivial. Therefore, we consider only the low distortion case, i.e. $D_{1}+D_{2}-D_{0} \leq 1$ 


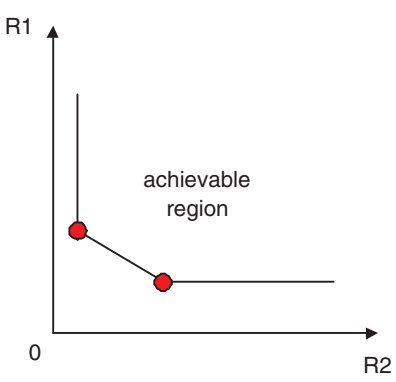

(a) achievable region

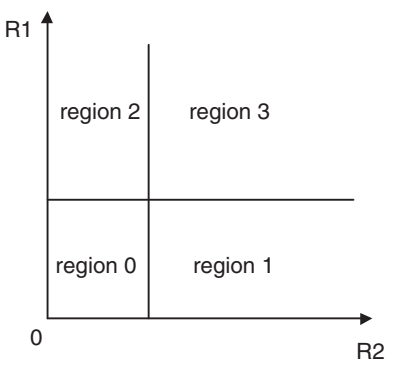

(c) regions with different utilities in 2 channels

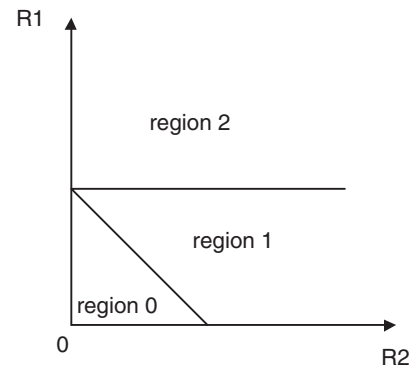

(b) regions with different utilities in single channel

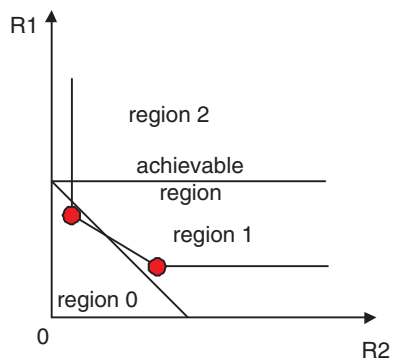

(d) optimal point in single channel
Fig. 3: Illustration of achievable region and regions with different utilities.

For fixed $D_{0}, D_{1}$ and $D_{2}$, it is easy to verify that the utility is different in different regions illustrated in Fig. 3 (b), i.e. the utility equals $U_{0}\left(D_{0}\right), U_{1}\left(D_{1}\right)$ and 0 in regions 0,1 and 2 , respectively. By comparing (a) and (b) in Fig. 3, it is easy to verify that the optimal expected utility is achieved by one of the corner points (e.g. the left corner point is optimal in Fig. 3 (d)). Then, we can use the procedure shown in Procedure 1 to search for the optimal distortions and rates exhaustively.

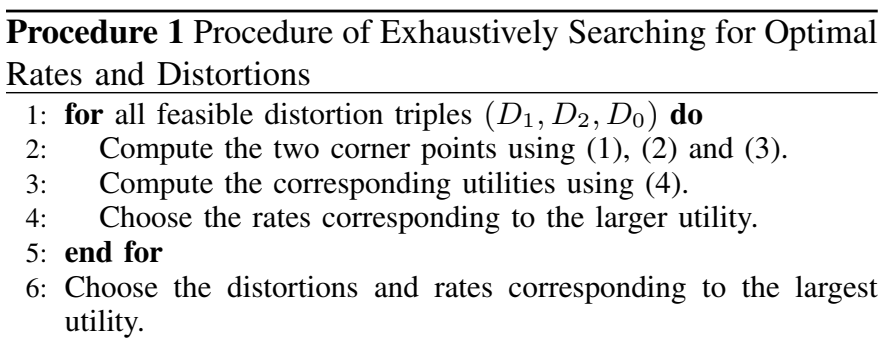

\section{MDC in Multiple Channels}

For multi-channel case, we consider two channels for simplicity. We denote by $P_{1 I}$ and $P_{2 I}$ the probabilities that channels 1 and 2 are idle, respectively (the two channels may not have the same parameters). Different from the single-channel case, we transmit the two descriptions over the two channels parallelly. Therefore, four possible events could happen:

- Event 0: both descriptions are received and distortion $D_{0}$ is achieved.

- Event 1: only description 1 is received and distortion $D_{1}$ is achieved.

- Event 2: only description 2 is received and distortion $D_{2}$ is achieved.
- Event 3: none of the descriptions is received.

The probabilities corresponding to the first three events are denoted by $P_{0}, P_{1}$ and $P_{2}$. Similarly to the single-channel case, we consider the following expected utility

$$
J=P_{1} U_{1}\left(D_{1}\right)+P_{1} U_{2}\left(D_{2}\right)+P_{0} U_{0}\left(D_{0}\right),
$$

where $U_{0}, U_{1}$ and $U_{2}$ are the utilities when events 0,1 and 2 happen, respectively.

It is easy to verify that, in the asymptotic case (the same definition as that in single-channel case), the probabilities $P_{0}$, $P_{1}$ and $P_{2}$ are given by

$$
P_{0}=\left\{\begin{array}{ll}
1, & \text { if } R_{1} \leq \alpha P_{1 I} \text { and } R_{2} \leq \alpha P_{2 I} \\
0, & \text { otherwise }
\end{array},\right.
$$

and

$$
P_{1}=\left\{\begin{array}{ll}
1, & \text { if } R_{1} \leq \alpha P_{1 I} \text { and } R_{2}>\alpha P_{2 I} \\
0, & \text { otherwise }
\end{array},\right.
$$

and

$$
P_{2}=\left\{\begin{array}{ll}
1, & \text { if } R_{1}>\alpha P_{1 I} \text { and } R_{2} \leq \alpha P_{2 I} \\
0, & \text { otherwise }
\end{array} .\right.
$$

The regions having different utilities are illustrated in Fig. 3 (d). Again, the optimal rates are obtained at one of the corner points of the achievable region. Therefore, we can use the same procedure as in Procedure 1 to search for the optimal distortions and rates.

\section{MDC FOR IMAGES}

For practical source coding of an $N_{1} \times N_{2}$ image, we use source splitting, which is a heuristic approach for MDC [6], to generate multiple descriptions. For compressing the image, we use two-dimensional discrete cosine transform (DCT) to convert the original signal to the DCT domain, i.e.

$$
\begin{aligned}
Y_{k_{1}, k_{2}} & =\sum_{n_{1}=0}^{N_{1}-1} \sum_{n_{2}=0}^{N_{2}-1} X_{n_{1}, n_{2}} \cos \left[\frac{\pi}{N_{1}}\left(n_{1}+\frac{\pi}{2}\right) k_{1}\right] \\
& \times \cos \left[\frac{\pi}{N_{2}}\left(n_{2}+\frac{\pi}{2}\right) k_{2}\right]
\end{aligned}
$$

where $Y_{k_{1}, k_{2}}$ is the signal in DCT domain and $X_{n_{1}, n_{2}}$ is the original image signal. Based on the DCT coefficients, we use different MDC approaches for single-channel case and twochannel case, respectively, which are illustrated in Fig. 4 (a) and (b). For simplicity, we consider only two descriptions.

\section{A. Single-Channel Case}

When there is only one single channel, we generate two successive descriptions. First, we sort the coefficients in the descending order of absolute values. Then, we encode the first $L_{1}$ coefficients into the first description and encode the following $L_{2}$ coefficients into the second description.

At the decoder, if only description 1 is successfully received, we use the $L_{1}$ DCT coefficients to reconstruct the image via inverse DCT by assuming that all other coefficients are zero. If both descriptions are received, we combine the $L_{1}+L_{2}$ DCT coefficients for image reconstruction. 


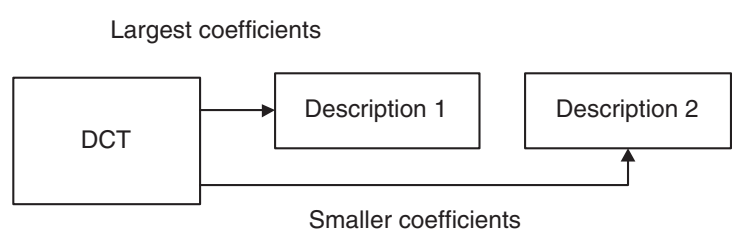

(a) encoder for single channel

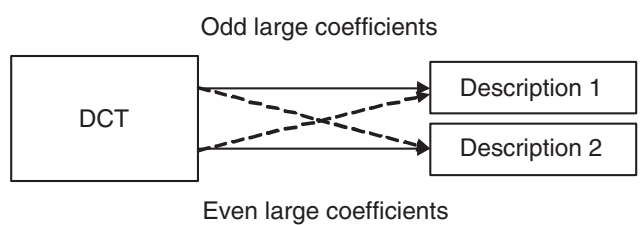

(b) encoder for two channels

Fig. 4: Encoder for images: (a) single channel; (b) two channels.

\section{B. Two-Channel Case}

When there are two channels, we generate two parallel descriptions. For simplicity, we assume that the two channels have the same parameters. Then, we consider the $L$ largest coefficients and encode the even and odd coefficients into descriptions 1 and 2 using $B_{h}$ bits for each coefficient. For introducing redundancy, we also add the even and odd coefficients into descriptions 2 and 1 using $B_{l}$ bits $\left(B_{l}<B_{h}\right)$ for each coefficient.

At the decoder, if only one of the descriptions is received before the deadline, we use the $L$ DCT coefficients (having different resolutions for even and odd ones) to reconstruct the original image via inverse DCT. If both descriptions are received before the deadline, the $L$ DCT coefficients having high resolution are used for the inverse DCT.

\section{Numerical Results}

In this section, we use numerical simulations to demonstrate the proposed algorithms of MDC for both Gaussian sources and image sources, respectively.

\section{A. MDC for Gaussian Source}

For Gaussian source, we use the model of Markov chain for primary user activity. The following utility function is used:

$$
U_{1}(D)=U_{0}(D)=\log \frac{1}{D} .
$$

We compute the optimal distortions and rates for different $\alpha$ (recall that $\alpha=\frac{T_{d}}{N}$, which represents the tolerance of delay) using the algorithm in Procedure 1. The optimal coding rates for the two successive descriptions transmitted over a single channel (with $P_{I}=0.9$ ) is shown in Fig. 5. We observe that the coding rate $R_{1}$ for the first description increases with $\alpha$ while the coding rate $R_{2}$ is not monotonic with $\alpha$. The optimal coding rates for the two parallel descriptions transmitted over two asymmetric channels (we assume $P_{1 I}=0.9$ and $P_{2 I}=$ 0.5 ) are plotted in Fig. 6. We observe that, when $\alpha$ is small, $R_{1}>R_{2}$ (high coding rate for the better channel); however,

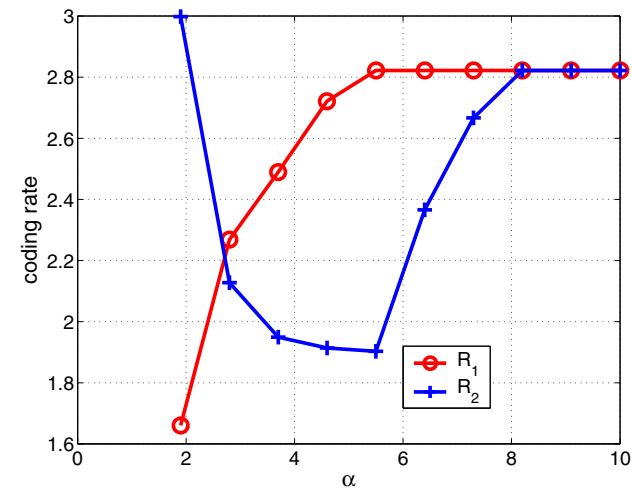

Fig. 5: Optimal coding rates for the single-channel case.

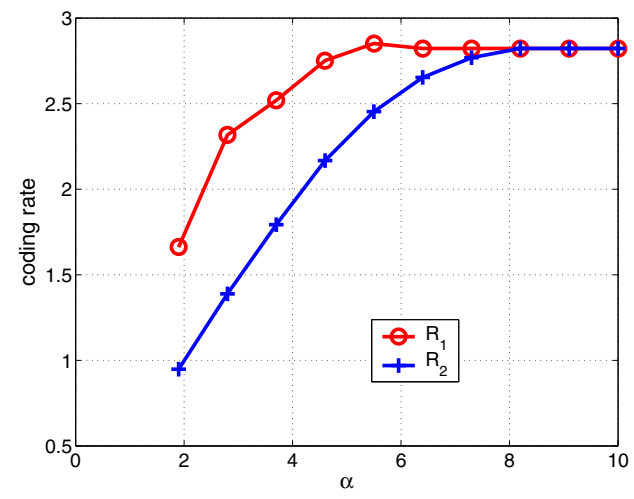

Fig. 6: Optimal coding rates for the two-channel case.

when $\alpha$ is sufficiently large, $R_{1}=R_{2}$, i.e. we should use the same coding rates for both channels.

\section{B. MDC for Images}

We use a semi-Markov chain to model the occupancy of primary users when studying the image source coding. For simplicity, we assume that the transition from idle to busy is instantaneous while it takes 1500 channel symbol periods to transit from busy to idle. We assume that $P_{I B}=0.001$ and $P_{B I}=0.1$. The deadline $T_{d}$ is set to $100 \mathrm{k}$ channel symbol periods. We assume that 16QAM is used as channel symbol (4 bits per symbol) such that the time (measured in the number of channel symbol periods) needed to transmit one description is given by $\frac{L B}{4}$, where $L$ is number of source symbols and $B$ is average number of bits used for each source symbol. If a $10 \mathrm{Mbps}$ transmission rate is guaranteed, the deadline is equivalent to $40 \mathrm{~ms}$. A $512 \times 512$ image 'Barbara' is used for testing.

1) Single-Channel Case: For a single channel, we set $L_{1}=2000$ and $L_{2}=3000$, using 16 bits for each source symbol. Since we need 18 bits to indicate the index of each DCT coefficients, $B$ is equal to 34 . Simulation shows that, with probability 0.285 , both descriptions can be received before the deadline, while only description 1 can be received with probability 0.715 (the probability that none of the descriptions is received is negligible). The reconstructed images using description 1 or both descriptions are shown in Fig. 7. We 


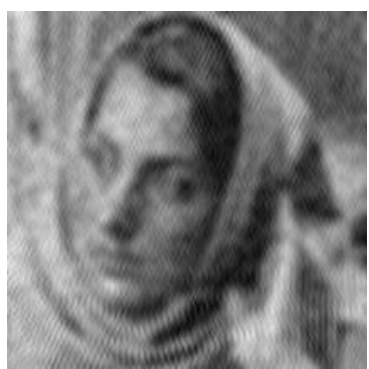

(a) image when both descriptions are received; probability: $28.5 \%$

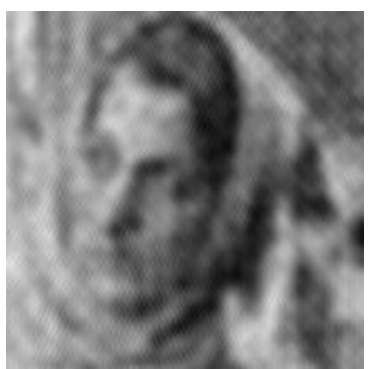

(b) image when description 1 is received; probability: $71.5 \%$
Fig. 7: Recovered images in a single cognitive radio channel.

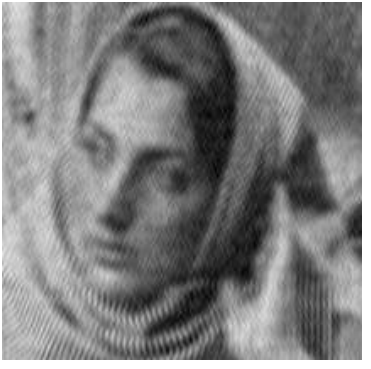

(a) image when both descriptions are received; probability: $57.7 \%$

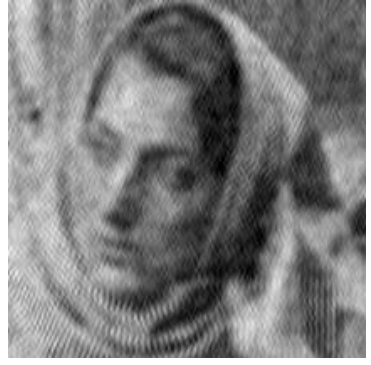

(b) image when only one description is received; probability: $36.4 \%$
Fig. 8: Recovered images with two cognitive radio channels.

observe that the image obtained from both descriptions is much better than that reconstructed from a single description. However, the reconstructed image is tolerable when only one description is received.

2) Two-Channel Case: When two channels exist, we set $L=6000$, i.e. using 6000 DCT coefficients for MDC. We use 24 bits to encode the even and odd coefficients for descriptions 1 and 2, respectively, while using 9 bits to encode the odd and even coefficients for descriptions 1 and 2, respectively (i.e. $B_{h}=24$ and $B_{l}=9$ ). Therefore, the average bits per source symbol, $B$, is given by $18+(24+9) / 2=34.5$. The reconstructed images are shown in Fig. 8. We observe that the image quality is slightly improved when both descriptions are received. We conjecture that, if a better combination is used (we simply combined the coefficients described using high resolution ( 24 bits) and discarded the descriptions with low resolution (9 bits)), the performance gain will be better.

\section{CONCLUSIONS}

We have applied MDC for source coding in cognitive radio systems to combat the interruptions from primary users when the data traffic is delay-sensitive and distortion tolerable. We have discussed the optimal selection of distortions and rates maximizing the expected utility for Gaussian source. For practical realtime image source coding, a simple technique of source splitting is applied for both single-channel and twochannel cases. Numerical results have demonstrated that the proposed MDC schemes work effectively under the interrup- tions in cognitive radio systems. We are expecting to extend the work to more source coding scenarios, e.g. video streaming in cognitive radio networks.

\section{ACKNOWLEDGEMENTS}

The author would like to thank Dr. Cong Shen in UCLA for the talk with him which motivates the study in this paper.

\section{REFERENCES}

[1] J. G. Apostolopoulos and M. D. Trott, "Path diversity for enhanced media streaming," IEEE Commun. Magazine, special issue on "Proxy Support for Streaming on the Internet”, Aug. 2001.

[2] T. Berger and Z. Zhang, "Minimum breakdown degradation in binary source coding," IEEE Trans. Inform. Theory, vol.29, pp.807-814, Nov. 1983.

[3] A. El Gamal and T. M. Cover, "Achievable rates for multiple descriptions," IEEE Trans. Inform. Theory, vol.28, pp.851-857, Nov. 1982.

[4] W. H. R. Equitz and T. M. Cover, "Successive refinement of information," IEEE Trans. on Inform. Theory, vol.37, pp.269-275, March 1991.

[5] A. Gahsemi and E. S. Sousa, "Collaborative spectrum sensing for opportunistic access in fading environment," in Proc. of IEEE DySPAN, Nov. 2005.

[6] V. K. Goyal, "Multiple description coding: Compression meets the network," IEEE Signal Processing Magazine, vol.18, pp.74-93, Sept. 2001.

[7] D. Hu, S. Mao and J. H. Reed, "On video multicast in cognitive radio networks," in Proc. of IEEE Conference on Computer Communications (Infocom), April 2009.

[8] W. Jiang and A. Ortega, "Multiple description coding via polyphase transform and selective quantization," in Proc. SPIE Conf. Visual Communication Image Processing, Feb. 1999.

[9] H. Kushwaha, Y. Xing, R. Chandramouli and H. Heffes, "Reliable multimedia transmission over cognitive radio networks using Fountain Codes," Proceedings of the IEEE, vol.96, no.1, Jan. 2008.

[10] S. Mao, X. Cheng, Y. Hou and H. Sherali, "Multiple description video multicast in wireless ad hoc networks," ACM/Kuwer Mobile Netw. Appl. J., vol.11, pp.63-73, Jan. 2006.

[11] J. Mitola, "Cognitive radio for flexible mobile multimedia communications," in Proc. IEEE Int. Workshop Mobile Multimedia Communications, pp. 3-10, 1999.

[12] B. Rimoldi, "Successive refinement of information: Characterization of the achievable rates," IEEE Trans. on Inform. Theory, vol.40, pp.253259, Jan. 1994.

[13] M. Schaar and F. Fu, "Spectrum access games and strategic learning in cognitive radio networks for delay-critical applications," Proceedings of The IEEE, vol.97, no.2, pp. 720-740, April 2009.

[14] H. P. Shiang and M. Scharr, "Queuing-based dynamic channel selection for hetorogeneous multimedia applications over cognitive radio networks," IEEE Trans. Multimedia, vol.10, pp. 896-909, Aug. 2008.

[15] G. Sun, U. Samarawickrama, J. Liang, C. Tian, C. Tu and T. D. Tran, "Multiple description coding with prediction compensation," IEEE Trans. Image Processing, vol.18, pp.1037-1047, Sept. 2001.

[16] R. Venkataramani, G. Kramer and V. K. Goyal, "Multiple description coding with many channels," IEEE Trans. on Inform. Theory, vo.49, pp.2106-2114, Sept. 2003.

[17] Y. Wang, A. R. Reibman and S. Lin, "Multiple description coding for video delivery," Proceedings of The IEEE, vol.93, pp.57-70, Jan. 2005.

[18] H. S. Witsenhausen, "On source networks with minimal breakdown degradation,” Bell Syst. Tech. J, vol.59, pp.1083-1087, July-Aug. 1980.

[19] R. Zamir, "Gaussian codes and Shannon bounds for multiple descriptions," IEEE Trans. on Inform. Theory, vol.45, pp.2629-2635, Nov. 1999.

[20] Q. Zhao and B. M. Sadler, "A survey of dynamic spectrum access," IEEE Signal Processing Magazine, vol. 24, pp. 79-89, May. 2007.

[21] R. Zheng, W. Zhuang and H. Jiang, "Scalable multiple description coding and distributed video streaming in $3 \mathrm{G}$ mobile communications," Wireless Communications and Mobile Computing, vol.5, pp.95-111, 2005. 